Case Report

\title{
Primary Tuberculosis of Middle Ear
}

\author{
Swati Tandon, Satinder Singh, Asish K.Lahiri and Shalabh Sharma
}

Sir Ganga Ram Hospital, Delhi, India

Correspondence should be addressed to: Swati Tandon; drswatitandon86@gmail.com

Received date: 3 February 2014; Accepted date: 4 July 2014; Published date: 26 September 2014

Academic Editor: Isamu Sugawara

Copyright @ 2014. Swati Tandon, Satinder Singh, Asish K.Lahiri and Shalabh Sharma. Distributed under Creative Commons CC-BY 3.0

\begin{abstract}
Primary Tuberculosis of the middle ear remains a diagnostic challenge due to unspecific and variable clinical presentations. A characteristic clinical triad of painless ear discharge, multiple perforations and pale granulations in middle ear cleft is often seen but maybe absent. We report the case of a 10 year old girl who presented with bilateral ear discharge with attic involvement and facial palsy to otolaryngology department of our hospital. Surgical findings revealed pale granulations in mastoid and middle ear. Histopathological and microbiological examination of excised tissue showed the presence of acid fast bacilli. There was no evidence of pulmonary or any other tubercular foci.
\end{abstract}

Keywords: tuberculous otitis media, tubercle, granuloma

\section{Introduction}

Tuberculosis is one of the major infectious diseases seen in developing countries like India. Tuberculous oto-mastoiditis was 1st described by Jean Louis Petit in 18th century $^{1}$. Wilde described the characteristic picture of painless ear discharge, multiple perforations and pale granulations in the middle ear cleft ${ }^{2}$. Incidence of TB otitis media has been reported as $0.04-0.9 \%$ in developed countries $^{3}$. It is usually secondary to infections in the lung, pharynx and nose. Here we present a case of primary tuberculous otitis media from our institution.

\section{Case Report}

A 10 year old girl presented to otolaryngology department of our hospital with bilateral ear discharge and hearing impairment since 3 years and left facial palsy of 20 days duration. She was a known case of Coeliac Disease on gluten free diet.

She had undergone right canal wall down mastoidectomy with facial nerve decompression on right side, the previous year in our institute following which her facial functions had improved completely. There was no history of ear ache or post auricular swelling or fever. There was no

Cite this Article as: Swati Tandon, Satinder Singh, Asish K.Lahiri and Shalabh Sharma (2014),"Primary Tuberculosis of Middle Ear ", International Journal of Case Reports in Medicine, Vol. 2014 (2014), 
history of tuberculosis in family or contact with TB. The child was using air conduction hearing aid in her right ear and used to visit hospital for periodic cleaning of right ear. On examination, the child was well nourished and afebrile. Otoscopic examination on left side revealed the presence of attic granulations with purulent discharge with exposed bone of external canal. Postoperative mastoid cavity with some pale granulations was seen on the right side. There was left grade IV lower motor neuron facial palsy (House-Brackman Staging). Pure Tone audiometry revealed mixed hearing loss bilaterally with air -bone gap of 50 decibels. Nerve Conduction studies were evident of severe motor axonal neuropathy of facial nerve on left side. Her systemic examination, pre-operative hematological investigations and chest X-Ray were normal. A High Resolution Computed Tomographic Scan of Temporal bone showed postoperative cavity on the right side with soft tissue attenuation in the left middle ear with sclerosis of mastoid and also sequestrum in the floor of left external canal (Fig.1).

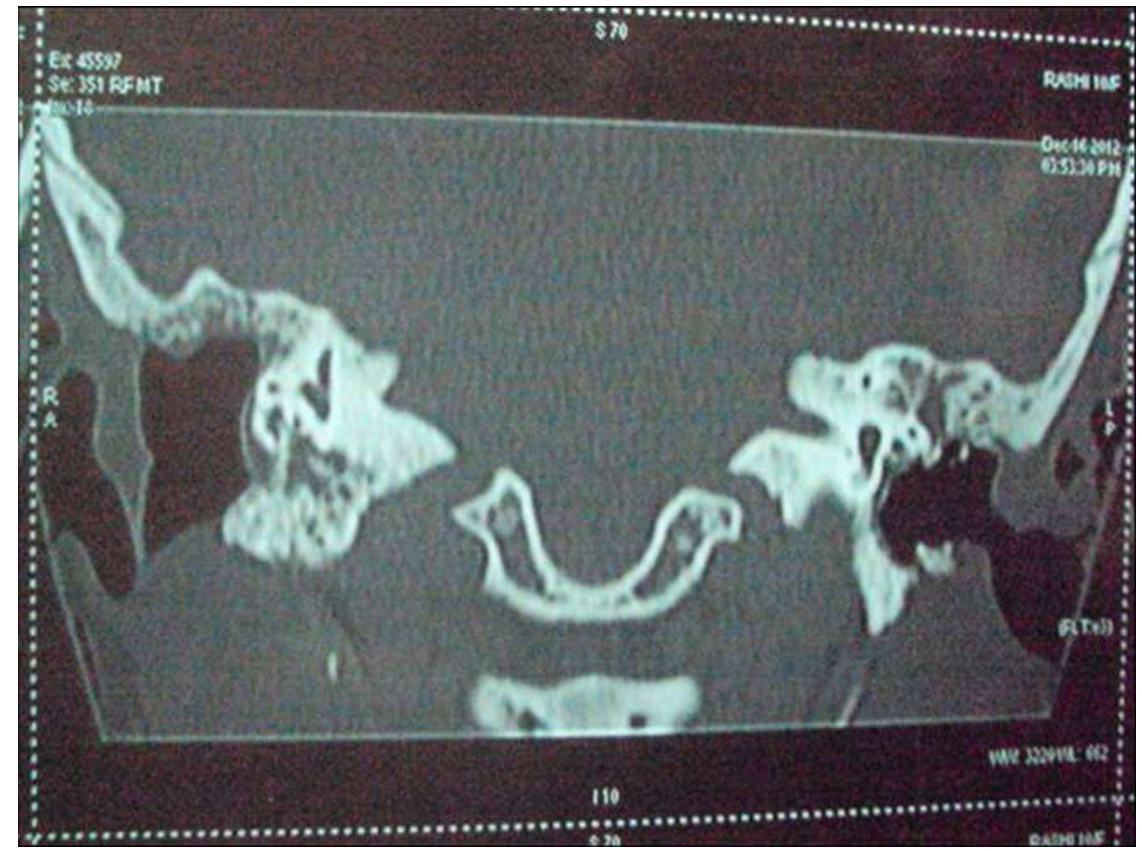

Figure1: Computed Tomographic Scan Of Temporal Bone Showing Right Mastoid Cavity and Soft Tissue Attenuation on Left Side

Based on the above findings, the child was taken up for left mastoidectomy and facial nerve exploration under general anaesthesia. Intra-operatively, pale relatively avascular granulations and necrotic bone were seen filling attic, antrum and mastoid. Incus and malleus were necrosed and unhealthy. Sequestrum from canal was removed. Fallopian canal was intact and was drilled to expose the nerve. Nerve Sheath in the horizontal segment was found edematous and thus incised to decompress the nerve. In view of the operative findings, granulation tissue was sent for histopathology and microbiological examination for AFB stain and culture. Histopathology revealed epithelioid granulomas with large areas of necrosis with numerous acid fast bacilli

Swati Tandon, Satinder Singh, Asish K.Lahiri and Shalabh Sharma (2014), International Journal of Case Reports in Medicine, DOI: 10.5171/2014.131557 
(Fig.2). Direct Fluorescence staining was also strongly positive for AFB (3+) (Fig.3). Rapid
AFB culture revealed Mycobacterium tuberculosis complex.

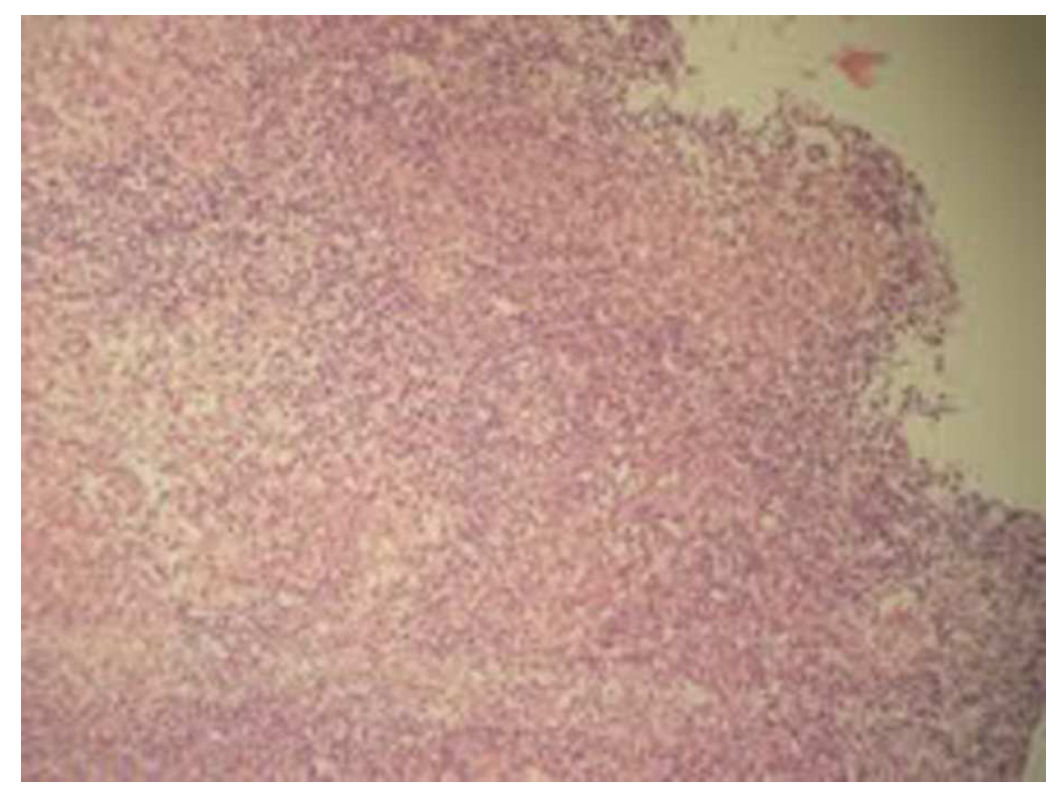

Figure 2: Epitheloid Granulomas Seen on Histopathology

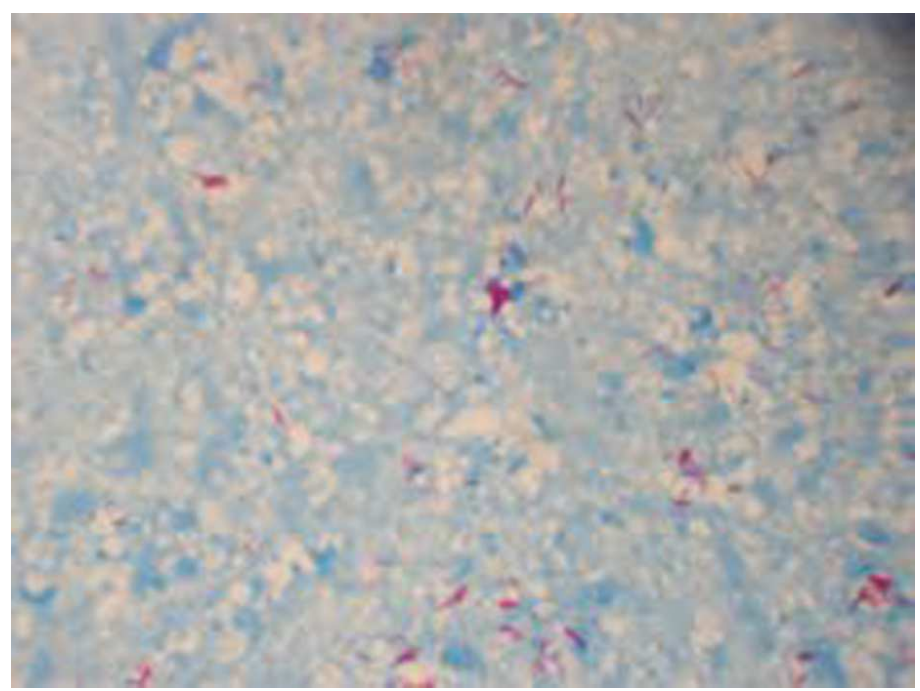

Figure 3: Acid Fast Bacilli Seen On Zeil Nielsen Staining

The child was started on category-1 Anti tubercular therapy which included four drug regimens of Rifampicin, Isoniazid, Pyrazinamide and Ethambutol in consultation with TB specialist. Facial nerve functions already showed improvement with return of muscle functions in the nasolabial fold and angle of mouth and better eye

Swati Tandon, Satinder Singh, Asish K.Lahiri and Shalabh Sharma (2014), International Journal of Case Reports in Medicine, DOI: 10.5171/2014.131557 
closure within 2 weeks of surgery. The presently child is on regular follow up with healing mastoid cavity.

\section{Discussion}

Primary tuberculosis of middle ear is a rare condition. TB otitis media should be suspected in cases of chronic otitis media when usual treatment is not producing desired results. The signs and symptoms are variable and nonspecific and thus difficult to differentiate from chronic non-TB otitis media. The classical symptoms of painless otorrhea, multiple tympanic membrane perforations and facial paralysis described in literature are usually not found. ${ }^{4}$

Uncommon and varied non-specific presentations usually result in delayed diagnosis. Infection reaches the middle ear through haematogenous route or ascending infection from Eustachian tube. The hearing loss is disproportionate to the clinical findings. The diagnosis is often made postoperatively or when complications such as facial paralysis, labyrinthitis, post auricular fistulae or abscess have occurred. The assessment should include a thorough examination of the ear, chest $x$-ray, and mantoux test. Bacteriological investigation of ear secretions may not be as sensitive, because of high rate of secondary bacterial infection that may interfere with the growth of Koch's bacillus. Chronic use of topical ear drops in such cases also interferes with culture sensitivity. Cultures on ear secretions are usually positive in around $20-30 \%$ of the cases ${ }^{4}$.

In our case, there was bilateral attic involvement similar to an unsafe atticoantral disease. Severity of disease coupled with facial palsy led us to operate on the right side. The intraoperative findings on the right side were cholesteatoma having no features so as to suspect tuberculosis.

Only intra-operative findings of the left side in which there were pale granulations with necrotic bone both in mastoid and in the external canal led us to suspect tuberculosis. The diagnosis of tuberculous otitis media was based on demonstration of acid fast bacilli in epithelioid granulomas on histopathology, with positive direct fluorescence staining. Positive AFB culture further confirmed the diagnosis.

Macroscopically, in TB otitis media, tubercles appear in the middle ear cavity affecting the mucosa over the promontory, the inner surface of the tympanic membrane and the mucosa of the antrum. These tubercles infiltrate with caseation and necrosis of bone. Granulations surrounding the ossicles result in absorption and destruction of ossicles ${ }^{5}$.

Microscopically, granulomas composed of epithelioid cells, lymphocytes and Langerhan's giant cells with central caseous necrosis, are seen on histopathological examination of tubercles. The 'caseating granulomas' are pathognomic histopathological feature of tuberculosis ${ }^{6}$. The inflammatory response is mediated by a type IV hypersensitivity reaction.

In literature, there have been few reports citing correlation between tuberculosis and celiac disease, but these are specific to gastrointestinal tuberculosis. There is no study in the literature correlating tuberculosis of middle ear with celiac disease. The correlation between tuberculosis and celiac disease may be related to malabsorption of vitamin $\mathrm{D}^{7}$. Vitamin D was recently shown to upregulate antimicrobial peptides such as LL-37, which are implicated in intracellular killing of Mycobacterium tuberculosis.13 Vitamin D can also induce nitric oxide synthase in macrophages, which again may suppress the growth of Mtuberculosis.14 An alternative possibility is that the HLA-DQ2 haplotype, which is strongly linked to coeliac disease, is associated with specific alleles of HLA class I and II molecules, as well as with genes for tumour necrosis factor (TNF) $\alpha$ and complement factors $\mathrm{C} 2$ and C4. TNF $\alpha$ polymorphisms may regulate host responses 
in tuberculosis. It is difficult to correlate this association in our patient.

Anti-Tubercular therapy is the mainstay of treatment. There is a need for post-operative antituberculous chemotherapy which provides a rapid and definitive resolution of infectious process. Some authors suggest that surgical intervention is useful for managing complications such as facial palsy and removal of sequestrum and improve drainage ${ }^{8}$. Surgery combined with ATT drugs gives a good chance for healing with dry ear.

Based on literature search, we found only a single case report of primary tuberculosis of middle ear with features similar to our case with attic involvement and facial palsy9. Facial nerve palsy has been reported in cases of tuberculosis otitis media in approximately $16 \%$ of adult cases and $35 \%$ of pediatric cases $^{10}$.

Our case report calls attention to the nonspecific findings of primary tuberculous otitis media. Since our patient had been operated on one side with findings of cholesteatoma intraoperatively, therefore we did not suspect tuberculosis in the other ear. If atypical clinical features are seen in a case of chronic otits media, cultures of ear discharge should be taken repeatedly since culture for tuberculosis has a low yield. High degree of suspicion is required for diagnosing tubercular otitis media since early diagnosis and prompt treatment can prevent ear damage and complications.

\section{References}

1.Granato L and Limae Silva LA. Tuberculous otitis media. Rev Bras Otolaryngol 1973;39: 125-32.

2.Skolnik PR, Nadol Jr JB and Baker AN. Tuberculous of the middle ear: Review of the literature with an instructive case report. Rev Infect Dis 1986;8: 403-10.

3.Weiner GM, O Connell JE, Pahor AL. The role of surgery in tuberculosis mastoiditis:
Appropriate chemotherapy is not always enough. J Laryngol Otol 1977;111: 752-3.

4. Mercêdes Fabiana Araújo, Thaís Gonçalves Pinheiro, Igor Teixeira Raymundo,

Vítor Yamashiro Rocha Soares, Pedro Ivo Machado Araújo, Ricardo Luiz de Melo Martins,Carlos Augusto Costa P Oliveira. Tuberculous Otitis Media. Int. Adv. Otol. 2011; 7:(3) 413-417

5. J F Birrell. Aural Tuberculosis in Children. Proc. roy. Soc. Med. Volume 66 April 1973

6.Alakananda

Dasgupta, Navjeevan Singh, and Arati Bhatia.Abdominal tuberculosis:a histopathological study with special refernce to intestinal perforation and mesenteric vasculopathy. J Lab Physicians. 2009 Jul-Dec; 1(2): 56-61

7. DS Sanders,J West,M K B Whyte.Coeliac disease and risk of tuberculosis: a population based cohort study. Thorax. Jan 2007; 62(1): $1-2$.

8. Myerson MC, Gilbert JG. Tuberculosis of the middle ear and mastoid. Arch Otolaryngol 1941;33: 231-250

9. Adhikari et al. Tuberculous otitis media with facial palsy. Nepalese Journal of ENT Head and Neck surgery 2010;17-18

10.Singh B. Role of surgery in tuberculosis mastoiditis. J Laryngo Otol 1991;105: 907-15 